\title{
Complex biliary stones management: cholangioscopy versus papillary large balloon dilation - a randomized controlled trial
}

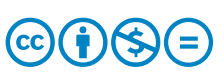

\author{
Authors \\ Tomazo Franzini, Renata Nobre Moura, Priscilla Bonifácio, Gustavo Oliveira Luz, Thiago Ferreira de Souza, Marcos \\ Eduardo Lera dos Santos, Gustavo Luis Rodela, Edson Ide, Paulo Herman, André Luis Montagnini, Luiz Augusto \\ Carneiro D’Albuquerque, Paulo Sakai, Eduardo Guimarães Hourneaux de Moura
}

\author{
Institution \\ University of Sao Paulo Medical School - Department of \\ Gastroenterology, Sao Paulo, Brazil \\ submitted 18.1.2017 \\ accepted after revision 25.10.2017 \\ Bibliography \\ DOI https://doi.org/10.1055/s-0043-122493 | \\ Endoscopy International Open 2018; 06: E131-E138 \\ (C) Georg Thieme Verlag KG Stuttgart · New York \\ ISSN 2364-3722 \\ Corresponding author \\ Tomazo Franzini, MD, PhD, Av. Dr. Enéas de Carvalho \\ Aguiar, 255 - Prédio dos Ambulatórios Pinheiros, \\ 05403-000 Sao Paulo, SP - Brazil \\ tomazof@uol.com.br
}

\section{ABSTRACT}

Background and study aims Endoscopic removal of biliary stones has high success rates, ranging between $85 \%$ to $95 \%$. Nevertheless, some stones may be challenging and different endoscopic methods have evolved. Papillary large balloon dilation after sphincterotomy is a widely used tech- nique with success rates ranging from 68 to $90 \%$ for stones larger than $15 \mathrm{~mm}$. Cholangioscopy allows performing lithotripsy under direct biliary visualization, either by laser or electrohydraulic waves, which have similar success rate (80\%-90\%). However, there is no study comparing these 2 techniques.

Patients and methods From April 2014 to June 2016, 100 patients were enrolled and randomized in 2 groups, using a non-inferiority hypothesis: cholangioscopy+electrohydraulic lithotripsy (group 1) and endoscopic papillary large balloon dilation (group 2). The main outcome was complete stone removal. Adverse events were documented. Mechanical lithotripsy was not performed. Failure cases had a second session with crossover of the methods.

Results The mean age was 56 years. 74 (75.5\%) patients were female. The initial overall complete stone removal rate was $74.5 \%$ (77.1\% in group 1 and $72 \%$ in group $2, P>$ 0.05). After second session the overall success rate achieved $90.1 \%$. Procedure time was significantly lower in group 2,-25.2 $\min (\mathrm{Cl} 95 \%-12.48$ to -37.91$)$. There were no significant differences regarding technical success rate, radiologic exposure and adverse events.

Conclusion Single-operator cholangioscopy-guided lithotripsy and papillary large balloon dilation are effective and safe approaches for removing complex biliary stones.

\section{Introduction}

Endoscopic retrograde cholangiopancreatography (ERCP) is the gold standard treatment of biliary duct stones [1,2] with success rate between $85 \%$ to $95 \%$ [3-8]. In experienced hands, it results in few adverse events (AEs), the most common of which are acute pancreatitis ( $1.3 \%$ to $6.7 \%$ ), infection ( $0.6 \%$ to $5 \%$ ), bleeding $(0.3 \%$ to $2.0 \%)$ and perforation $(0.1 \%$ to $1.1 \%)[8,9]$.

However, some biliary stones may become a challenge, depending on their location, number and especially dimensions. While there is no consensus regarding the definition of complexity criteria, difficulties may arise due to local and anatomi- cal conditions such as strictures, diverticula, disproportion between the size of distal bile duct and the stone (difference greater than $2 \mathrm{~mm}$ ), post-surgical altered anatomy and multiple (10 or more) or large stones (with a diameter of $\geq 15 \mathrm{~mm}$ ) $[1,8,10-13]$.

Many endoscopic techniques have evolved to manage difficult biliary stones. Mechanical lithotripsy (ML) is an established technique, with high success rates after multiple sessions, however some cases can be challenging regarding stone and common bile duct ( $C B D$ ) diameter, as well as time-consuming and with several possible AEs [5 - 8]. Endoscopic papillary large balIoon dilation (EPLBD) after sphincterotomy was first described 
by Ersoz et al in 2003 [14] with success rate varying between $68 \%$ and $90 \%$ for stones $\geq 15 \mathrm{~mm}$ [15-19]. EPLBD has been shown as a safe and effective method, with low complication rates [20].

The Spyglass Direct Visualization System ${ }^{\mathrm{TM}}$ (Boston Scientific, Inc., USA) is a single-operator cholangioscope that enables target endobiliary therapies without the need of large biliary dilation and sphincterotomies, which can reduce possible AEs. Cholangioscopy-guided electrohydraulic lithotripsy (EHL) allows the fragmentation of difficult biliary stones and its subsequent removal [21], generating sparks directly on the stone, minimizing the risk of biliary wall damage, with success rates reported between $80 \%$ and $90 \%[22,23]$.

Although some studies have shown the effectiveness and safety of cholangioscopy-guided EHL for the treatment of biliary stones, $[22,24,25]$ the lack of randomized controlled trials (RCT) comparing this endoscopic modality with EPLBD justifies this research, aiming to evaluate these 2 methods regarding complete stone removal, occurrence of adverse events and the association of techniques, optimizing the endoscopic approach to difficult biliary stones.

\section{Patients and methods}

\section{Study design}

We prospectively enrolled 100 patients in a RCT performed at the Endoscopy Unit of University of São Paulo Medical School Clinics Hospital from April 2014 to June 2016. Ethical approval was granted by the institutional review board. All participants provided written informed consent. This study was registered at World Health Organization (WHO) primary basis under the number RBR-5wx47j. All procedures were performed by 2 investigators (TAPF, EGHM), both with considerable experience in ERCP (over 350 procedures/year).

\section{Patients and sample size}

Inclusion criteria included age 18 years or older and presence of difficult biliary stones defined in our study as multiple (more than 10), size greater than $15 \mathrm{~mm}$, presence of disproportion between the stone and distal common bile duct (greater than $2 \mathrm{~mm}$ ) or biliary stricture with a stone upstream. Exclusion criteria were pregnancy, patients who underwent gastrointestinal surgery or liver transplant previously and acute cholangitis.

Accordingly with our hypothesis of non-inferiority between the methods under analysis, sample size was based on their success rates reported in the literature, ranging from $73 \%$ to $91 \%$ after multiple sessions $[18,19,22,23]$. As this study was designed to have a crossover process if initial failure, we had to consider that in both groups the success could range from lowest to higher rate. Therefore, we expected to find a success rate of approximately $91 \%$. A non-inferiority margin of $17 \%$ was considered, so in the worst-case scenario the success rate would be $74 \%$, after the 2 sessions. Considering that, to obtain tests with $5 \%$ significance and $80 \%$ power, 45 cases would be necessary in each group.

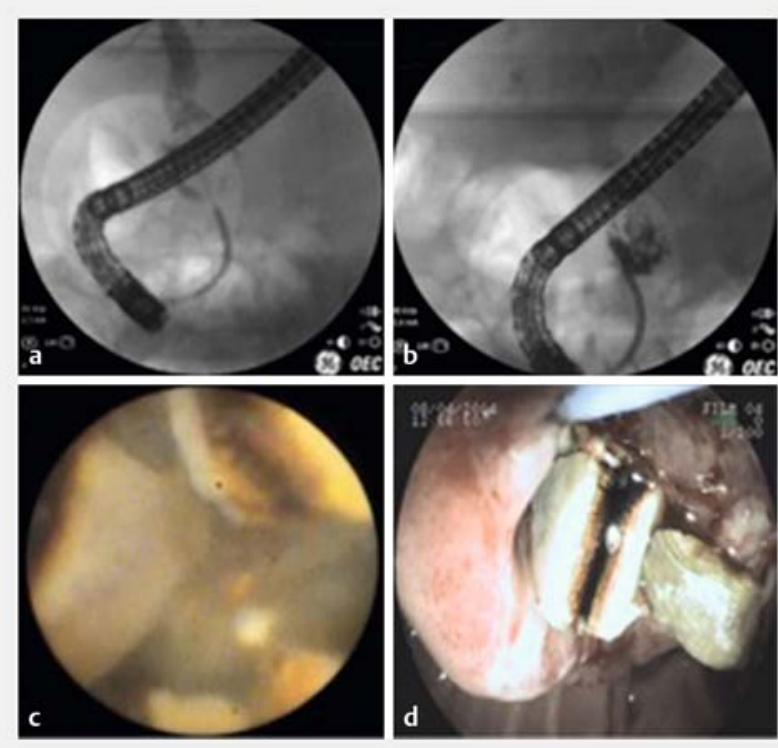

- Fig. 1 Group 1 endoscopic approach. a Large stone under fluoroscopy view. b Post-EHL fractured stone. c Cholangioscopy view of fractured stone. $\mathbf{d}$ Stone fragments removal.

Foreseeing a possible loss of follow-up of $10 \%$, the ideal sample was 50 cases in each group. Therefore, 100 cases were necessary for the development of this study.

\section{Randomization}

Randomization was performed using a computer-generated system (Urbaniak, GC, \& Plous, S. [2013]: Research Randomizer (Version 4.0) [Computer software], which was put into use on 03/18/2014, www.randomizer.org/). During the ERCP procedure, after cholangiography confirmed presence of a complex biliary stone, conventional techniques were performed in an attempt to remove the stone and clear the common bile duct (CBD). When these methods failed, the eligible patients were randomly assigned in 2 groups: single-operator cholangioscopy $(\mathrm{SOC})+$ electrohydraulic lithotripsy (group 1) and endoscopic papillary large balloon dilation (group 2).

\section{Interventions}

SOC was performed with the first-generation of SpyGlass ${ }^{\mathrm{TM}}$ platform in group 1. With this system, a delivery catheter (SpyScope ${ }^{\mathrm{TM}}$ ) is introduced through the duodenoscope into the biliary tree under a guidewire. After proper positioning, the lithotripter fiber is used with a generator with a 70 - to 100-watt power output, depending on the response of stone fragmentation. After fracture, the stones were retrieved using a Dormia basket and/or a retrieval balloon catheter ( $\triangleright$ Fig. 1).

In group 2, after sphincterotomy, a balloon dilator was passed over a guidewire and positioned across the ampullary site. Balloon diameter was determined depending on the diameter of CBD, stone size and presence of strictures. Inflation was performed with distilled water and contrast until maximum biliary duct caliber or after complete disappearance of radiologic 

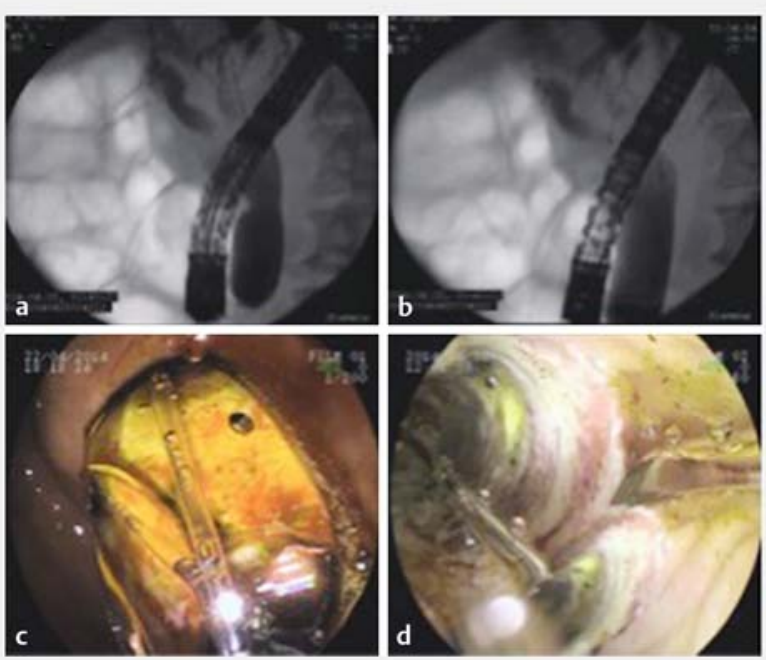

- Fig. 2 Group 2 endoscopic approach. a Radiologic waist. b Balloon dilation with waist disappearance. $\mathbf{c}$ Endoscopic view of large balloon dilation of papilla. $\mathbf{d}$ Balloon "scope" viewing segment under dilation.

waist. Balloon was kept inflated for 3 minutes to avoid bleeding. Reinflation was performed for the same time if hemorrhage was noted. After dilation, stones were retrieved using a Dormia basket and/or a retrieval balloon catheter ( $>$ Fig. 2).

In both groups, in case of failure, either biliary drainage with plastic stents or crossover to the other method was performed immediately. Patients who received a plastic stent were scheduled for crossover in a second attempt. This study design was intended to analyze if or when the methods under investigation could be associated with an improved success rate.

Introduction of plastic biliary stent due to partial biliary clearance and/or need to crossover was considered as failure of the initial approach. Mechanical lithotripsy was not performed in any patient. Ciprofloxacin, $400 \mathrm{mg}$ IV, was given as prophylaxis to all patients in both groups.

\section{Outcomes}

The primary outcome of the study was complete stone removal after 2 sessions with different techniques under analysis, if needed. Secondary end points included AEs, procedure time and $x$-ray exposure time.

AEs such as acute cholangitis, perforation, acute pancreatitis and bleeding, were classified according to time of diagnosis (immediately during the procedure, 24 hours later or 1 week later) and also according to severity (mild, moderate or severe), relation with the technique (definitely related, possibly related, not assigned), treatment (drugs, endoscopic or surgery) and outcome (recovered without sequelae, recovered with sequelae or death).

Cholangitis was defined as abdominal pain, fever, chills and/ or jaundice. Pancreatitis was defined as persistent abdominal pain and vomiting associated with a serum amylase level more than 3 times the upper limit, which required hospitalization or an extension of it. Perforation was considered if disruption of duodenal or biliary wall, was identified under direct endoscopic visualization, or with fluoroscopy evidence of contrast medium extravasation or by imaging tests. Bleeding was defined as occurrence of papillary or biliary bleed after the endoscopic procedure that requiring transfusion or interventions.

\section{Statistical analysis}

For quantitative variables, statistical data were presented as minimum and maximum values and calculation of means, standard deviations and median. For qualitative data, absolute and relative frequencies were calculated. A Student $t$ test was used to compare means between groups. When data were not taken as normal value, we used the nonparametric Mann-Whitney test. Comparisons involving categorical data were made with the chi-square test or Fisher's exact test.

A $P$ value $<0.05$ was regarded as statistically significant.

\section{Results}

\section{Patients}

From April 2014 to June 2016, 2302 ERCPs were performed at our endoscopy unit. Of these, 1841 patients (79.97\%) had bile duct stones. 100 patients (5.4\%) with difficult stones that failed standard removal techniques met the inclusion criteria and were enrolled in our study. 2 patients in group 1 were excluded due to different diagnosis, verified during cholangioscopy ( Fig.3).

The mean age in group 1 was 52.13 years and 59.76 years in group $2(P=0.032)$. There was no statistically significant difference between the 2 groups in terms of gender, symptoms, previous endoscopic procedures and stones characteristics, as number, size, shape and location. Patients' baseline characteristics and outcomes are summarized in > Table1.

\section{Study outcomes}

Stone removal was complete in $73(74.5 \%)$ of the 98 patients during the first session. The per-protocol (PP) analysis of initial success rate was not significantly different between the 2 groups after this first attempt (37/48 patients [77.1\%] vs. 36/ 50 patients [72\%], $P>0.05$, respectively); with a $95 \%$ confidence interval $(\mathrm{Cl})-12.13 \%$ to $22.29 \%$.

Procedure time was statistical different between the groups, with 72.3 minutes in group 1 and 47.1 minutes in group 2, -25.2 min (CI 95\%-12.48 to - 37.91). X-ray exposure time was not significantly different between the 2 groups.

Stone fragmentation after 1 session of EHL was complete in 35 patients $(72.9 \%)$, partial in 10 patients $(20.8 \%)$ and unsuccessful in 3 (6.2\%).

Regarding the 25 overall cases of failure, 20 (80\%) returned to perform another ERCP as a crossover process. In group 1, there were 11 failures, 1 (2.1\%) was lost to follow-up and 10 (20.8\%) were submitted to a second ERCP session. Of these, 10 patients had endoscopic papillary large balloon dilation performed, in 7 (70\%) of whom the procedure failed and in $3(30 \%)$ of whom it was a success. 


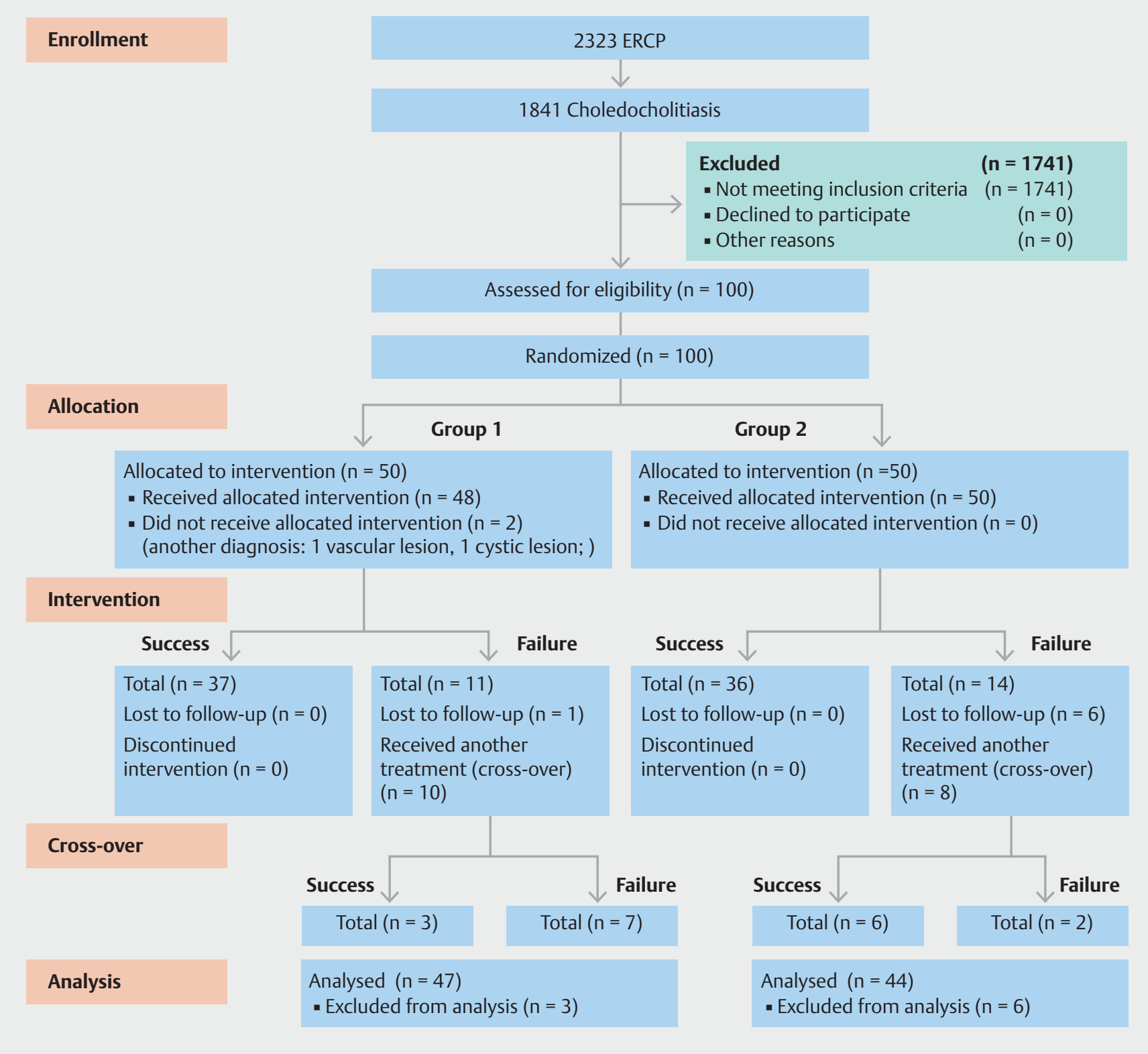

Fig. 3 Flow diagram of study.

In group 2, there were 14 cases of failure, 4 (8\%) were lost to follow-up and 2 (4\%) chose to receive surgical treatment. 8 patients $(16 \%)$ had undergone a second session, with success in 6 (75\%) after SOC + EHL.

After the second session, the overall PP success rate increased from $74.5 \%$ to $90.1 \%$ with no significant difference between the 2 groups (40/47 patients [85.1\%] vs. $42 / 44$ patients [95.4\%], $P>0.05$, respectively); with a $95 \%$ confidence interval (CI) $-22.24 \%$ to $1.546 \%$. Therefore we proposed an evidencebased algorithm for management of large and disproportional biliary stones ( $\triangleright$ Fig. 4 ).

The rates of AEs were not significantly different between the 2 groups (2/48 [4.2\%] vs. 6/50 [12\%], $P>0.05)$. In group 1, 1 patient $(2.1 \%)$ had acute cholangitis, and another had acute pancreatitis, both of mild intensity, and the recovered after standard clinical treatment. In group 2, 1 patient (2\%) had periampullary laceration, 1 (2\%) distal CBD perforation, 2 (4\%) bleeding, and 2 (4\%) acute pancreatitis. All of the cases were treated conservatively with good recovery. There were no late complications (after 7 days).

\section{Discussion}

According to results of this trial, endoscopic papillary large balloon dilation and single-operator cholangioscopic-guided electrohydraulic lithotripsy are equally effective and safe procedures for endoscopic removal of difficult biliary stones in which a standard approach with sphincterotomy and extraction balloon has failed. Our data support the hypothesis of non-inferiority, and overall rates of technical success after first and second sessions were not significantly different between the 2 
- Table 1 Patient characteristics.

\begin{tabular}{|c|c|c|c|}
\hline Variable & Group 1 & Group 2 & $P$ value \\
\hline Age (years) & $52.13 \pm 17.64$ & $59.76 \pm 16.99$ & 0.032 \\
\hline Gender (M/F) & Nov-37 & $13 / 37$ & 0.723 \\
\hline \multicolumn{4}{|l|}{ Previous procedures } \\
\hline - Cholecystectomy & 34 & 27 & 0.086 \\
\hline - ERCP & 43 & 38 & 0.076 \\
\hline - Sphincterotomy & 43 & 38 & 0.076 \\
\hline - Balloon dilation & 10 & 7 & 0.372 \\
\hline - Plastic stent & 31 & 35 & 0.068 \\
\hline - Duration of stent (weeks) & $3,34 \pm 3,69$ & $3,35 \pm 4,92$ & 0.441 \\
\hline \multicolumn{4}{|l|}{ Stones } \\
\hline \multicolumn{4}{|l|}{ - Number } \\
\hline$-<3$ & $31(64.6 \%)$ & $38(76 \%)$ & \multirow[t]{2}{*}{0.216} \\
\hline-3 & $17(35.4 \%)$ & $12(24 \%)$ & \\
\hline \multicolumn{4}{|l|}{ - Size } \\
\hline$-<15 \mathrm{~mm}$ & $9(18.8 \%)$ & $9(18 \%)$ & \multirow[t]{3}{*}{0.995} \\
\hline$-15-20 \mathrm{~mm}$ & $18(37.5 \%)$ & $19(38 \%)$ & \\
\hline$-20 \mathrm{~mm}$ & $21(43.8 \%)$ & $22(44 \%)$ & \\
\hline \multicolumn{4}{|l|}{ - Shape } \\
\hline - Rounded & $20(41.7 \%)$ & $12(24 \%)$ & 0.062 \\
\hline - Faceted & $20(41.7 \%)$ & $20(40 \%)$ & 0.867 \\
\hline - Longitudinal & $8(16.7 \%)$ & $19(38 \%)$ & 0.018 \\
\hline \multicolumn{4}{|l|}{ Associated conditions } \\
\hline - Biliary stricture & $1(10.4 \%)$ & 3 & \\
\hline - Duodenal diverticulum & $5(2.1 \%)$ & 3 & 0.865 \\
\hline - Stone-choledochal disproportion & 16 & 20 & \\
\hline - First session technical success rate & $37(77.1 \%)$ & $36(72 \%)$ & 0.930 \\
\hline - Procedure time (min) & $72.3 \pm 33.95$ & $47.1 \pm 29.37$ & $<0.001$ \\
\hline - X-ray exposure (min) & $10.85 \pm 6.95$ & $9.73+6.61$ & 0.371 \\
\hline \multicolumn{4}{|l|}{ Adverse events } \\
\hline - Acute pancreatitis & $1(2.1 \%)$ & $2(4 \%)$ & \\
\hline - Acute cholangitis & $1(2.1 \%)$ & 0 & \\
\hline - Bleeding & 0 & $2(4 \%)$ & \\
\hline - Laceration & 0 & $1(2 \%)$ & \\
\hline " Perforation & 0 & $1(2 \%)$ & \\
\hline $\begin{array}{l}\text { - Overall per protocol technical success rate after second ses- } \\
\text { sion (crossover) }\end{array}$ & $40 / 47(85.1 \%)$ & $42 / 44(95.4 \%)$ & 0.1147 \\
\hline
\end{tabular}




\section{EPLBD}

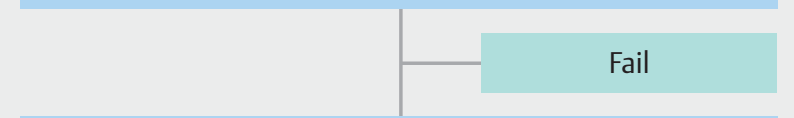

Associate ML (regarding stone and CBD size and expertise)

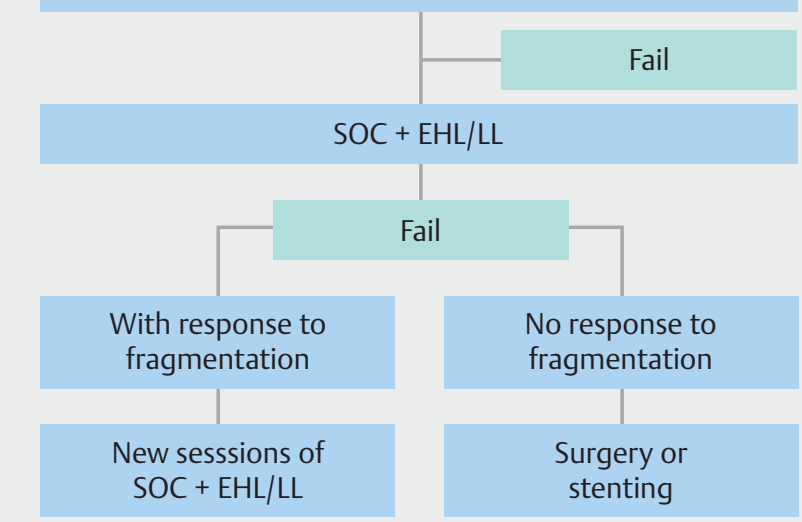

- Fig. 4 Proposed evidence-based algorithm for management of large and disproportional biliary stones.

groups $(77.1 \%$ vs. $72 \%, P>0.05)$ ( $95 \%$ confidence interval $(\mathrm{Cl})$ $12.13 \%$ to $22.29 \%$ ), and ( $85.1 \%$ vs. $95.4 \%, P>0.05$ ); ( $95 \%$ confidence interval $(\mathrm{Cl})-22.24 \%$ to $1.546 \%$ ), respectively. After completion of 2 sessions with the different techniques in the study, the overall PP success rate was $90.1 \%$, which is compatible with literature reports.

The majority of patients in this study were female $(75.5 \%)$ and the average age was 56.02 years with a standard deviation of 17.64 years and a median of 57 years. Despite randomization, the mean ages between groups 1 and 2 showed a significant difference ( 52.13 vs. 59.76, $P=0.032$ ).

Ersoz et al. [14] first reported the use of large-diameter $(12-20 \mathrm{~mm})$ balloon dilation after sphincterotomy as an alternative for managing difficult bile duct stones. They reported an $83 \%$ success rate and an overall complication rate of $15 \%$, including a $3 \%$ pancreatitis rate. The rationale for this method is to facilitation of retrieval of large stones without the need of ML and reduced risk of procedure-related complication. Mechanical lithotripsy can be effective in experienced hands, although limitations are reported in large and multiple stones and when there is lack of space between stones and bile duct wall $[2,15$, $16,17,26,27]$. Stefanidis et al. [20] reported a higher rate of AEs with mechanical lithotripsy, especially cholangitis, when compared with endoscopic papillary large-balloon dilation. In our study mechanical lithotripsy was not associated with any case, because it could have influenced our objectives to evaluate, in an isolated way, the safety and effectiveness of each of the evaluated methods, as well as their association with outcomes. Nevertheless, in our unit as well as in most centers, mechanical lithotripsy is currently used for large stones when the CBD can be dilated enough to allow sufficient space to the lithotripter opening and stone capture.
Some authors have demonstrated the efficacy and safety of endoscopic papillary large-balloon dilation after single or multiple sessions, with success rates ranging from $70 \%$ to $95 \%$, complication rates from $1 \%$ to $12 \%$ and need for mechanical lithotripsy in $10 \%$ of patients $[10,12,20,28-31]$.

Our study showed a success rate with this method of $72 \%$ (36/50 patients) in a single initial session. Endoscopic papillary large-balloon dilation, besides being widely available, is an easy method to teach and learn. The correct positioning of the balloon, the use of water-soluble contrast solution to verify the radiological waist disappearance, the slow and gradual application of pressure in the balloon, as well as special attention to not lose the guidewire position are important technical details that should be respected. When endoscopic papillary large-balloon dilation was performed after crossover in a second session, success was achieved in $3 / 10$ patients $(30 \%)$ who had already failed a first attempt with cholangioscopy plus electrohydraulic lithotripsy.

The complication rate in the endoscopic papillary large-balloon dilation group was $12 \%(6 / 50)$. There were 2 patients (4\%) who developed mild post-endoscopic retrograde cholangiopancreatography pancreatitis, all of whom recovered well after conservative management. 2 patients presented with bleeding, both with marked distal biliary disproportion, as observed in other reports. Endoscopic treatment with adrenaline solution injection was successful and there was no need for blood transfusions.

The most feared complication of endoscopic papillary largeballoon dilation is perforation. When faced with patients with distal bile duct stricture or long distal segment of disproportion, it is prudent to review the indication for this approach and alternatives should be considered, [32] including lithotripsy. To minimize the risk of perforation, the size of the dilated balloon should not exceed the size of the CBD. In this study, there was 1 case of deep laceration and 1 perforation, both immediately treated with success. Laceration was sealed with an endoclip and CBD perforation was treated with a fully covered metal stent which was inserted and removed after 3 weeks. Post-crossover, endoscopic papillary large-balloon dilation presented no AEs.

Regarding cholangioscopic-guided electrohydraulic lithotripsy, previous studies report success rates ranging from $73 \%$ to $91 \%[23,33,34]$. In a multicenter study, Chen et al. [24] reported 66 patients that were treated with cholangioscopy plus lithotripsy with a $71 \%$ success rate after a single session, and stone response to fragmentation achieved in $92 \%$. There were $6.1 \% \mathrm{AEs}$, including 2 cases of acute cholangitis (3.3\%). Kalaitzakis et al. [34] performed 165 single-operator cholangioscopies, $35(21 \%)$ with EHL. The successful stone removal rate in the first session was $73 \%$, and acute cholangitis was the most common AE $4.8 \%$ (8/165 cases), with 1 death.

However, few RCTs have been published. In 2009, Seelhoff et al. [35] showed success in removing biliary stones in 17/22 (77\%) patients after 1 cholangioscopy session. A recent publication reported the use of single-operator cholangioscopy in 148 patients, 31 cases of electrohydraulic lithotripsy for stones with an average size of $20.6 \mathrm{~mm}$. There were $23(74.2 \%)$ cases 
of success, with $9.67 \%, 58.06 \%, 3.22 \%$ and $3.22 \%$ in the first, second, fourth and eighth sessions, respectively. AEs occurred in $5.4 \%$ (8/148 cases), 4 (2.70\%) cholangitis, 2 (1.35\%) pancreatitis, 1 (0.67\%) bleeding, and $1(0.67 \%)$ aspiration. [36]

Our study showed a success rate of $77.1 \%$ in the first session and 2 AEs (4.2\%): 1 acute pancreatitis (2.1\%) and 1 acute cholangitis (2.1\%), both with favorable outcome after conservative treatment. There was also a favorable response to electrohydraulic lithotripsy in 45 of 48 patients (93.7\%); complete in 35 (72.9\%), partial in $10(20.8 \%)$ and none in $3(6.2 \%)$ patients. These data suggest that patients who had a partial response to electrohydraulic lithotripsy could benefit from new sessions with the same method, however, this cannot be justified in cases with no response.

When cholangioscopy-guided lithotripsy was performed after crossover (endoscopic papillary large-balloon dilation failure cases) in a second session, success was achieved in 6/8 patients (75\%), and no AEs were noted. This finding reinforces the proposed algorithm of inserting cholangioscopy-guided lithotripsy as an additional step in therapeutic endoscopy.

This study had 2 exclusions in group 1 after initial randomization. Patients with suspected complex choledicolithiasis after ERCP, which was not confirmed by cholangioscopy direct visualization. In 1 patient, an extrinsic and pulsatile lesion compressing the CBD was seen. At the same time, a doppler endoscopic ultrasound was carried out and showed the presence of blood flow, compatible with varice. In the other case, the filling defect was due to a cystic lesion prominent inside the CBD. In both patients, biliary plastic stents were introduced. These cases of exclusion reinforce findings from other studies in which cholangioscopy was clearly able to correctly identify the etiology of filling defects $[37,38]$.

Time of procedure was significantly higher in group 1 (72.3 vs. 47.1 minutes, $-25.2 \mathrm{~min}$ (C195\%-12.48 to - 37.91), reflecting the higher complexity of cholangioscopy plus an electrohydraulic lithotripsy procedure. It is also important to highlight that the SpyGlass platform used in our study was the first generation, nowadays called "Legacy," a fiber optic-based system. Currently a digital system version, "SpyGlass DS," is available, providing better images, irrigation and ergonomics, which could improve lithotripsy and significantly decrease total procedure time and radiation exposure. Current data on use of both techniques following the methods in this study suggest that endoscopic papillary large-balloon dilation should be the primary approach for complex biliary stones after failure of standard techniques. Nevertheless, when the first session did not provide resolution, patients received crossover to the other group method, resulting in overall success rates above $90 \%$. Therefore, a second endoscopic attempt should always be made, and a lithotripsy technique would be preferable. It is reasonable, as well, to affirm that single-operator cholangioscopyguided lithotripsy is suitable for difficult stones with distal strictures or disproportion that causes limitation on dilation. In the other hand, endoscopic papillary large-balloon dilation can be effective for multiple and large stones without strictures and with a compatible diameter of distal CBD.
Limitations of this study are the impossibility of blinding the investigators to the methods used in each patient, absence of mechanical lithotripsy association as well as being a single-center RCT.

\section{Conclusion}

Single-operator cholangioscopic electrohydraulic lithotripsy as well as endoscopic papillary large balloon dilation are effective and safe techniques for endoscopic treatment of complex biliary stones, with high success rates and low incidence of $A E$.

The association of the methods demonstrates that they can be complementary to each other, with a consequent improvement in biliary clearance. Based on this evidence, a therapeutic algorithm with this approach is suggested, providing 1 more step when conventional endoscopic management has failed.

\section{Competing interests}

None

\section{References}

[1] Bergman JJ, Rauws EAJ, Tijssen JGP et al. Biliary endoprostheses in elderly patients with endoscopically irretrievable common bile duct stones: report on 117 patients. Gastrointest Endosc 1995; 42: 195 201

[2] Horiuchi A, Nakayama Y, Kajiyama M et al. Biliary stenting in the management of large or multiple common bile duct stones. Gastrointest Endosc 2010; 71: 1200-1203.e2

[3] Cotton PB. Endoscopic management of bile duct stones (apples and oranges). Gut 1984; 25: 587-597

[4] Cairns SR, Dias L, Cotton PB et al. Additional endoscopic procedures instead of urgent surgery for retained common bile duct stones. Gut 1989; 30: 535- 540

[5] Sivak MV. Endoscopic management of bile duct stones. Am J Surg 1989; 158: $228-240$

[6] Sherman S, Hawes RH, Lehman GA. Management of bile duct stones. Semin Liver Dis 1990; 10: 205-221

[7] Cipoletta L, Costamagna G, Bianco MA et al. Endoscopic mechanical lithotripsy of difficult bile duct stones. Br J Surg 1997; 84: 1407- 1409

[8] Bergman JJ, Rauws EA, Fockens P et al. Randomised trial of endoscopic balloon dilation versus endoscopic sphincterotomy for removal of bile duct stones. Lancet 1997; 349: 1124-1129

[9] Freeman ML, Nelson DB, Sherman S et al. Complications of endoscopic biliary sphincterotomy. N Engl J Med 1996; 335: 909-918

[10] Attasaranya S, Cheon YK, Vittal H et al. Large- diameter biliary orifice balloon dilation to aid in endoscopic bile duct stone removal: a multicenter series. Gastrointest Endosc 2008; 67: 1046- 1052

[11] Sharma SS, Jain P. Should we redefine large common bile duct stone? World J Gastroenterol 2008; 14: 651-652

[12] Heo J, Kang D, Jung HJ et al. Endoscopic sphincterotomy plus largeballoon dilation versus endoscopic sphincterotomy for removal of bile- duct stones. Gastrointest Endosc 2007; 66: 720-726

[13] Kim HJ, Choi HS, Park JH et al. Factors influencing the technical difficulty of endoscopic clearance of bile duct stones. Gastrointest Endosc 2007; 66: $1154-1160$ 
[14] Ersoz G, Tekesin O, Ozutemiz AO et al. Biliary sphincterotomy plus dilation with a large balloon for bile duct stones that are difficult to extract. Gastrointest Endosc 2003; 57: 156 - 159

[15] Schneider MU, Matek W, Bauer R et al. Mechanical lithotripsy of bile duct stones in 209 patients-effect of technical advances. Endoscopy 1988; 20: $248-253$

[16] Shaw MJ, Mackie RD, Moore JP et al. Results of a multicenter trial using a mechanical lithotripter for the treatment of large bile duct stones. Am J Gastroenterol 1993; 88: 730 - 733

[17] Chang WH, Chu CH, Wang TE et al. Outcome of simple use of mechanical lithotripsy of difficult common bile duct stones. World J Gastroenterol 2005; 11: 593-596

[18] Maydeo A, Bhandari S. Balloon sphincteroplasty for removing difficult bile duct stones. Endoscopy 2007; 39: 958 - 961

[19] Draganov PV, Evans W, Fazel A et al. Large size balloon dilation of the ampulla after biliary sphincterotomy can facilitate endoscopic extraction of difficult bile duct stones. J Clin Gastroenterol 2009; 43: $782-786$

[20] Stefanidis G, Viazis N, Pleskow D et al. Large balloon dilation vs. mechanical lithotripsy for the management of large bile duct stones: a prospective randomized study. Am J Gastroenterol 2011; 106: 278 285

[21] Chen YK, Pleskow DK. SpyGlass single-operator peroral cholangiopancreatoscopy system for the diagnosis and therapy of bile-duct disorders: a clinical feasibility study (with video). Gastrointest Endosc 2007; 65: 832-841

[22] Arya N, Nelles SE, Haber GB et al. Electrohydraulic lithotripsy in 111 patients: a safe and effective therapy for difficult bile duct stones. Am J Gastroenterol 2004; 99: 2330-2334

[23] Maydeo A, Kwek BE, Bhandari S et al. Single-operator cholangioscopyguided laser lithotripsy in patients with difficult biliary and pancreatic ductal stones (with videos). Gastrointest Endosc 2011; 74: 1308 1314

[24] Chen YK, Parsi MA, Binmoeller KF et al. Single-operator cholangioscopy in patients requiring evaluation of bile duct disease or therapy of biliary stones (with videos). Gastrointest Endosc 2011; 74: 805-814

[25] Moura EG, Franzini T, Moura RN et al. Cholangioscopy in bile duct disease: a case series. Arq Gastroenterol 2014; 51: 250 - 254

[26] Trikudanathan G, Navaneethan U, Parsi MA. Endoscopic management of difficult common bile duct stones. World J Gastroenterol 2013; 19 : $165-173$
[27] Yasuda I, Itoi T. Recent advances in endoscopic management of difficult bile duct stones. Dig Endosc 2013; 25: 376 - 385

[28] Bang S, Kim MH, Park JY et al. Endoscopic papillary balloon dilation with large balloon after limited sphincterotomy for retrieval of choledocholithiasis. Yonsei Med J 2006; 47: 805-810

[29] Minami A, Hirose S, Nomoto T et al. Small sphincterotomy combined with papillary dilation with large balloon permits retrieval of large stones without mechanical lithotripsy. World J Gastroenterol 2007; 13: $2179-2182$

[30] Misra SP, Dwivedi M. Large-diameter balloon dilation after endoscopic sphincterotomy for removal of difficult bile duct stones. Endoscopy 2008; 40: 209-213

[31] Attam R, Freeman ML. Endoscopic papillary large balloon dilation for large common bile duct stones. J Hepatobiliary Pancreat Surg 2009; 16: $618-623$

[32] Lee DK, Jahng JH. Alternative methods in the endoscopic management of difficult common bile duct stones. Dig Endosc 2010; 22: S79-S84

[33] Seelhoff A, Schumacher B, Neuhaus H. Single operator peroral cholangioscopic guided therapy of bile duct stones. J Hepatobiliary Pancreat Sci 2011; 18: 346 - 349

[34] Kalaitzakis E, Webster G], Oppong KW et al. Diagnostic and therapeutic utility of single-operator peroral cholangioscopy for indeterminate biliary lesions and bile duct stones. Eur J Gastroenterol Hepatol 2012; 24: 656-664

[35] Seelhoff A, Schumacher B, Neuhaus H. Prospective study of SpyGlass guided laser lithotripsy of bile duct stones after failure of conventional endoscopic techniques. Gastrointest Endosc 2009; 69: AB261

[36] Kurihara T, Yasuda I, Isayama $\mathrm{H}$ et al. Diagnostic and therapeutic single- operator cholangiopancreatoscopy in biliopancreatic diseases: Prospective multicenter study in Japan. World J Gastroenterol 2016; 22: $1891-1901$

[37] Fukuda Y, Tsuyuguchi T, Sakai Y et al. Diagnostic utility of peroral cholangioscopy for various bile-duct lesions. Gastrointest Endosc 2005; 62: $374-382$

[38] Farrell J], Bounds BC, Al-Shalabi S et al. Single-operatorduodenoscope-assisted cholangioscopy is an effective alternative in the management of choledocholithiasis not removed by conventional methods, including mechanical lithotripsy. Endoscopy 2005; 37: 542 - 547 